\title{
One of the Odd Zeta Values from $\zeta(5)$ to $\zeta(25)$ Is Irrational. By Elementary Means
}

Wadim ZUDILIN

Department of Mathematics, IMAPP, Radboud University, PO Box 9010, 6500 GL Nijmegen, The Netherlands

E-mail:w.zudilin@math.ru.nl

URL: http://www.math.ru.nl/ wzudilin/

Received January 31, 2018, in final form March 26, 2018; Published online March 29, 2018

https://doi.org/10.3842/SIGMA.2018.028

\begin{abstract}
Available proofs of result of the type 'at least one of the odd zeta values $\zeta(5), \zeta(7), \ldots, \zeta(s)$ is irrational' make use of the saddle-point method or of linear independence criteria, or both. These two remarkable techniques are however counted as highly nonelementary, therefore leaving the partial irrationality result inaccessible to general mathematics audience in all its glory. Here we modify the original construction of linear forms in odd zeta values to produce, for the first time, an elementary proof of such a result a proof whose technical ingredients are limited to the prime number theorem and Stirling's approximation formula for the factorial.
\end{abstract}

Key words: irrationality; zeta value; hypergeometric series

2010 Mathematics Subject Classification: 11J72; 11M06; 33C20

\section{Introduction}

Without touching deeply a history of the question (see [3] for an excellent account of this), we notice that the irrationality of the zeta values — values of Riemann's zeta function

$$
\zeta(s)=\sum_{n=1}^{\infty} \frac{1}{n^{s}}
$$

at integers $s=2,3, \ldots$, is known for even $s$ and also for $s=3$, while there are only partial results in this direction for odd $s \geq 5$. A starting point here has been set in the work [1] with further development, particularly focusing on $\zeta(5)$, in [7] and [10].

We fix an odd integer $s \geq 7$. Our strategy is constructing two sequences of linear forms $r_{n}$ and $\hat{r}_{n}$ living in the $\mathbb{Q}$-space $\mathbb{Q}+\mathbb{Q} \zeta(3)+\mathbb{Q} \zeta(5)+\cdots+\mathbb{Q} \zeta(s)$, for which we have a control of the common denominators $\lambda_{n}$ of rational coefficients and an elementary access to their asymptotic behaviour as $n \rightarrow \infty$; more importantly, the two coefficients of $\zeta(3)$ in these forms are proportional (with factor 7), so that $7 r_{n}-\hat{r}_{n}$ belongs to the space $\mathbb{Q}+\mathbb{Q} \zeta(5)+\cdots+\mathbb{Q} \zeta(s)$. Finally, using $7 r_{n}-\hat{r}_{n}>0$ and the asymptotics $\lambda_{n}\left(7 r_{n}-\hat{r}_{n}\right) \rightarrow 0$ as $n \rightarrow \infty$ of the linear forms

$$
\lambda_{n}\left(7 r_{n}-\hat{r}_{n}\right) \in \mathbb{Z}+\mathbb{Z} \zeta(5)+\mathbb{Z} \zeta(7)+\cdots+\mathbb{Z} \zeta(s)
$$

when $s=25$, we conclude that it cannot happen that all the quantities $\zeta(5), \zeta(7), \ldots, \zeta(25)$ are rational.

This paper is a contribution to the Special Issue on Orthogonal Polynomials, Special Functions and Applications (OPSFA14). The full collection is available at https://www.emis.de/journals/SIGMA/OPSFA2017.html 
The original idea of using the so-called well-poised hypergeometric series to construct linear forms in zeta values of a given parity is due to Ball and Rivoal [1]; our new ingredient here is using simultaneously such series and their 'twists by half', for an appropriate choice of the parameters. More precisely, our hypergeometric series assume the form

$$
r_{n}=\sum_{\nu=1}^{\infty} R_{n}(\nu) \quad \text { and } \quad \hat{r}_{n}=\sum_{\nu=1}^{\infty} R_{n}\left(\nu-\frac{1}{2}\right),
$$

where the rational-function summand $R_{n}(t)$ is defined as follows:

$$
\begin{aligned}
R(t)=R_{n}(t) & =\frac{n !^{s-5} \prod_{j=1}^{n}(t-j) \cdot \prod_{j=1}^{n}(t+n+j) \cdot 2^{6 n} \prod_{j=1}^{3 n}\left(t-n-\frac{1}{2}+j\right)}{\prod_{j=0}^{n}(t+j)^{s}} \\
& =\frac{2^{6 n} n !^{s-5} \prod_{j=0}^{6 n}\left(t-n+\frac{1}{2} j\right)}{\prod_{j=0}^{n}(t+j)^{s+1}} .
\end{aligned}
$$

The following Sections 2 and 3 discuss, respectively, the arithmetic and analysis of the forms (1). In Section 4 we use this information to conclude with the proof of the claimed result and make some relevant comments.

\section{Arithmetic ingredients}

The notation $d_{n}$ will be used for the least common multiple of $1,2, \ldots, n$. Recall that the prime number theorem is equivalent to the asymptotics

$$
\lim _{n \rightarrow \infty} d_{n}^{1 / n}=e .
$$

A rational function $S(t)$ of the form

$$
S(t)=\frac{P(t)}{\left(t-t_{1}\right)^{s_{1}}\left(t-t_{2}\right)^{s_{2}} \cdots\left(t-t_{q}\right)^{s_{q}}},
$$

whose denominator has degree larger than its numerator, possesses a unique partial-fraction decomposition

$$
S(t)=\sum_{j=1}^{q} \sum_{i=1}^{s_{j}} \frac{b_{i, j}}{\left(t-t_{j}\right)^{i}} .
$$

The coefficients here can be computed on the basis of explicit formula

$$
b_{i, j}=\left.\frac{1}{\left(s_{j}-i\right) !}\left(S(t)\left(t-t_{j}\right)^{s_{j}}\right)^{\left(s_{j}-i\right)}\right|_{t=t_{j}}
$$

for all $i, j$ in question. This procedure can be illustrated on the following examples, in which all the exponents $s_{j}$ are equal to 1 :

$$
\begin{aligned}
& \frac{n !}{\prod_{j=0}^{n}(t+j)}=\sum_{k=0}^{n} \frac{(-1)^{k}\left(\begin{array}{c}
n \\
k
\end{array}\right)}{t+k}, \\
& \frac{\prod_{j=1}^{n}(t-j)}{\prod_{j=0}^{n}(t+j)}=\sum_{k=0}^{n} \frac{(-1)^{n+k}\left(\begin{array}{c}
n+k \\
n
\end{array}\right)\left(\begin{array}{c}
n \\
k
\end{array}\right)}{t+k}, \\
& \frac{\prod_{j=1}^{n}(t+n+j)}{\prod_{j=0}^{n}(t+j)}=\sum_{k=0}^{n} \frac{(-1)^{k}\left(\begin{array}{c}
2 n-k \\
n
\end{array}\right)\left(\begin{array}{l}
n \\
k
\end{array}\right)}{t+k},
\end{aligned}
$$




$$
\begin{aligned}
& \frac{2^{2 n} \prod_{j=1}^{n}\left(t+\frac{1}{2}-j\right)}{\prod_{j=0}^{n}(t+j)}=\sum_{k=0}^{n} \frac{(-1)^{n+k}\left(\begin{array}{c}
2 n+2 k \\
2 n
\end{array}\right)\left(\begin{array}{c}
2 n \\
n+k
\end{array}\right)}{t+k}, \\
& \frac{2^{2 n} \prod_{j=1}^{n}\left(t-\frac{1}{2}+j\right)}{\prod_{j=0}^{n}(t+j)}=\sum_{k=0}^{n} \frac{\left(\begin{array}{c}
2 k \\
k
\end{array}\right)\left(\begin{array}{c}
2 n-2 k \\
n-k
\end{array}\right)}{t+k}, \\
& \frac{2^{2 n} \prod_{j=1}^{n}\left(t+n-\frac{1}{2}+j\right)}{\prod_{j=0}^{n}(t+j)}=\sum_{k=0}^{n} \frac{(-1)^{k}\left(\begin{array}{c}
4 n-2 k \\
2 n
\end{array}\right)\left(\begin{array}{c}
2 n \\
k
\end{array}\right)}{t+k} .
\end{aligned}
$$

It also means that the function $R(t)$ in $(2)$ can be written as

$$
R(t)=\sum_{i=1}^{s} \sum_{k=0}^{n} \frac{a_{i, k}}{(t+k)^{i}}
$$

with the recipe to compute the coefficients $a_{i, k}$ in its partial-fraction decomposition. At the same time, the function $R(t)$ is a product of 'simpler' rational functions given above, with all coefficients of their partial fractions being integral.

Lemma 1. Let $k_{1}, \ldots, k_{q}$ be pairwise distinct numbers from the set $\{0,1, \ldots, n\}$ and $s_{1}, \ldots, s_{q}$ positive integers. Then the coefficients in the expansion

$$
\frac{1}{\prod_{j=1}^{q}\left(t+k_{j}\right)^{s_{j}}}=\sum_{j=1}^{q} \sum_{i=1}^{s_{j}} \frac{b_{i, j}}{\left(t+k_{j}\right)^{i}}
$$

satisfy

$$
d_{n}^{s-i} b_{i, j} \in \mathbb{Z}, \quad \text { where } i=1, \ldots, s_{j} \quad \text { and } \quad j=1, \ldots, q,
$$

where $s=s_{1}+\cdots+s_{q}$.

In particular,

$$
d_{n}^{s-i} a_{i, k} \in \mathbb{Z}, \quad \text { where } i=1, \ldots, s \quad \text { and } \quad k=0,1, \ldots, n,
$$

for the coefficients in (4).

Proof. Denote the rational function in question by $S(t)$. The statement is trivially true when $q=1$, therefore we assume that $q \geq 2$. In view of the symmetry of the data, it is sufficient to demonstrate the inclusions (5) for $j=1$. Differentiating a related product $m$ times, for any $m \geq 0$, we obtain

$$
\begin{aligned}
\frac{1}{m !}\left(S(t)\left(t+k_{1}\right)^{s_{1}}\right)^{(m)} & =\frac{1}{m !}\left(\prod_{j=2}^{q}\left(t+k_{j}\right)^{-s_{j}}\right)^{(m)}=\sum_{\substack{\ell_{2}, \ldots, \ell_{q} \geq 0 \\
\ell_{2}+\cdots+\ell_{q}=m}} \prod_{j=2}^{q} \frac{1}{\ell_{j} !}\left(\left(t+k_{j}\right)^{-s_{j}}\right)^{\left(\ell_{j}\right)} \\
& =\sum_{\substack{\ell_{2}, \ldots, \ell_{q} \geq 0 \\
\ell_{2}+\cdots+\ell_{q}=m}} \prod_{j=2}^{q}(-1)^{\ell_{j}}\left(\begin{array}{c}
s_{j}+\ell_{j}-1 \\
\ell_{j}
\end{array}\right)\left(t+k_{j}\right)^{-\left(s_{j}+\ell_{j}\right)} .
\end{aligned}
$$

This implies that

$$
b_{i, 1}=\sum_{\substack{\ell_{2}, \ldots, \ell_{q} \geq 0 \\
\ell_{2}+\cdots+\ell_{q}=s_{1}-i}} \prod_{j=2}^{q}(-1)^{\ell_{j}}\left(\begin{array}{c}
s_{j}+\ell_{j}-1 \\
\ell_{j}
\end{array}\right) \frac{1}{\left(k_{j}-k_{1}\right)^{s_{j}+\ell_{j}}}
$$


for $i=1, \ldots, s_{1}$. Using $d_{n} /\left(k_{j}-k_{1}\right) \in \mathbb{Z}$ for $j=2, \ldots, q$ and $\sum_{j=2}^{q}\left(s_{j}+\ell_{j}\right)=s-i$ for each individual summand, we deduce the desired inclusion in (5) for $j=1$, hence for any $j$.

The second claim in the lemma follows from considering $R(t)$ as a product of the 'simpler' rational functions.

Lemma 2. For the coefficients $a_{i, k}$ in (4), we have

$$
a_{i, k}=(-1)^{i-1} a_{i, n-k} \quad \text { for } \quad k=0,1, \ldots, n \quad \text { and } \quad i=1, \ldots, s,
$$

so that

$$
\sum_{k=0}^{n} a_{i, k}=0 \quad \text { for } i \text { even. }
$$

Proof. Since $s$ is odd, the function (2) possesses the following (well-poised) symmetry: $R(-t-$ $n)=-R(t)$. Substitution of the relation into (4) results in

$$
\begin{aligned}
-\sum_{i=1}^{s} \sum_{k=0}^{n} \frac{a_{i, k}}{(t+k)^{i}} & =\sum_{i=1}^{s} \sum_{k=0}^{n} \frac{a_{i, k}}{(-t-n+k)^{i}}=\sum_{i=1}^{s}(-1)^{i} \sum_{k=0}^{n} \frac{a_{i, k}}{(t+n-k)^{i}} \\
& =\sum_{i=1}^{s}(-1)^{i} \sum_{k=0}^{n} \frac{a_{i, n-k}}{(t+k)^{i}},
\end{aligned}
$$

and the identities in the lemma follow from the uniqueness of decomposition into partial fractions. The second statement follows from

$$
\sum_{k=0}^{n} a_{i, k}=(-1)^{i-1} \sum_{k=0}^{n} a_{i, n-k}=(-1)^{i-1} \sum_{k=0}^{n} a_{i, k} .
$$

Lemma 3. For each n,

$$
r_{n}=\sum_{\substack{i=2 \\ i \text { odd }}}^{s} a_{i} \zeta(i)+a_{0} \quad \text { and } \quad \hat{r}_{n}=\sum_{\substack{i=2 \\ i \text { odd }}}^{s} a_{i}\left(2^{i}-1\right) \zeta(i)+\hat{a}_{0},
$$

with the following inclusions available:

$$
d_{n}^{s-i} a_{i} \in \mathbb{Z} \quad \text { for } \quad i=3,5, \ldots, s, \quad \text { and } \quad d_{n}^{s} a_{0}, d_{n}^{s} \hat{a}_{0} \in \mathbb{Z} .
$$

Notice that

$$
\left(2^{i}-1\right) \zeta(i)=\sum_{\ell=1}^{\infty} \frac{1}{\left(\ell-\frac{1}{2}\right)^{i}}
$$

for $i \geq 2$.

Proof. Our strategy here is to write the series in (1) using the partial-fraction decomposition (4) of $R(t)$. To treat the first sum $r_{n}$ we additionally introduce an auxiliary parameter $z>0$, which we later specialise to $z=1$ :

$$
\begin{aligned}
r_{n}(z) & =\sum_{\nu=1}^{\infty} R_{n}(\nu) z^{\nu}=\sum_{\nu=1}^{\infty} \sum_{i=1}^{s} \sum_{k=0}^{n} \frac{a_{i, k} z^{\nu}}{(\nu+k)^{i}} \\
& =\sum_{i=1}^{s} \sum_{k=0}^{n} a_{i, k} z^{-k} \sum_{\nu=1}^{\infty} \frac{z^{\nu+k}}{(\nu+k)^{i}}=\sum_{i=1}^{s} \sum_{k=0}^{n} a_{i, k} z^{-k}\left(\operatorname{Li}_{i}(z)-\sum_{\ell=1}^{k} \frac{z^{\ell}}{\ell^{i}}\right) \\
& =\sum_{i=1}^{s} \operatorname{Li}_{i}(z) \sum_{k=0}^{n} a_{i, k} z^{-k}-\sum_{i=1}^{s} \sum_{k=0}^{n} \sum_{\ell=1}^{k} \frac{a_{i, k} z^{-(k-\ell)}}{\ell^{i}}
\end{aligned}
$$


where

$$
\operatorname{Li}_{i}(z)=\sum_{\ell=1}^{\infty} \frac{z^{\ell}}{\ell^{i}}
$$

for $i=1, \ldots, s$ are the polylogarithmic functions. The latter are well defined at $z=1$ for $i \geq 2$, where $\operatorname{Li}_{i}(1)=\zeta(i)$, while $\operatorname{Li}_{1}(z)=-\log (1-z)$ does not have a limit as $z \rightarrow 1^{-}$. By taking the limit as $z \rightarrow 1^{-}$in the above derivation and using $R_{n}(\nu)=O\left(\nu^{-2}\right)$ as $\nu \rightarrow \infty$, we conclude that

$$
\sum_{k=0}^{n} a_{1, k}=\lim _{z \rightarrow 1^{-}} \sum_{k=0}^{n} a_{1, k} z^{-k}=0,
$$

and

$$
r_{n}=\sum_{i=2}^{s} \zeta(i) \sum_{k=0}^{n} a_{i, k}-\sum_{i=1}^{s} \sum_{k=0}^{n} a_{i, k} \sum_{\ell=1}^{k} \frac{1}{\ell^{i}} .
$$

We proceed similarly for $\hat{r}_{n}$, omitting introduction of the auxiliary parameter $z$. Since $R(t)$ in (2) vanishes at $t=-\frac{1}{2},-\frac{3}{2}, \ldots,-n+\frac{1}{2}$, we can shift the starting point of summation for $\hat{r}_{n}$ to $t=-m-\frac{1}{2}$, where $m=\left\lfloor\frac{n-1}{2}\right\rfloor$, so that

$$
\begin{aligned}
\hat{r}_{n}= & \sum_{\nu=-m}^{\infty} R_{n}\left(\nu-\frac{1}{2}\right)=\sum_{\nu=-m}^{\infty} \sum_{i=1}^{s} \sum_{k=0}^{n} \frac{a_{i, k}}{\left(\nu+k-\frac{1}{2}\right)^{i}} \\
= & \sum_{i=1}^{s} \sum_{k=0}^{n} a_{i, k} \sum_{\nu=-m}^{\infty} \frac{1}{\left(\nu+k-\frac{1}{2}\right)^{i}} \\
= & \sum_{i=1}^{s} \sum_{k=0}^{m} a_{i, k} \sum_{\nu=-m}^{\infty} \frac{1}{\left(\nu+k-\frac{1}{2}\right)^{i}}+\sum_{i=1}^{s} \sum_{k=m+1}^{n} a_{i, k} \sum_{\nu=-m}^{\infty} \frac{1}{\left(\nu+k-\frac{1}{2}\right)^{i}} \\
= & \sum_{i=1}^{s} \sum_{k=0}^{m} a_{i, k}\left(\sum_{\ell=k-m}^{0} \frac{1}{\left(\ell-\frac{1}{2}\right)^{i}}+\sum_{\ell=1}^{\infty} \frac{1}{\left(\ell-\frac{1}{2}\right)^{i}}\right) \\
& +\sum_{i=1}^{s} \sum_{k=m+1}^{n} a_{i, k}\left(\sum_{\ell=1}^{\infty} \frac{1}{\left(\ell-\frac{1}{2}\right)^{i}}-\sum_{\ell=1}^{k-m-1} \frac{1}{\left(\ell-\frac{1}{2}\right)^{i}}\right) \\
= & \sum_{i=2}^{s}\left(2^{i}-1\right) \zeta(i) \sum_{k=0}^{n} a_{i, k}+\sum_{i=1}^{s} \sum_{k=0}^{m} a_{i, k} \sum_{\ell=0}^{m-k} \frac{(-1)^{i}}{\left(\ell+\frac{1}{2}\right)^{i}} \\
& -\sum_{i=1}^{s} \sum_{k=m+1}^{n} a_{i, k} \sum_{\ell=1}^{k-m-1} \frac{1}{\left(\ell-\frac{1}{2}\right)^{i}} .
\end{aligned}
$$

Now the statement of the lemma follows from the representations in (7) and (8), Lemma 2, the inclusions (6) of Lemma 1 and

$$
\begin{array}{ll}
d_{n}^{i} \sum_{\ell=1}^{k} \frac{1}{\ell^{i}} \in \mathbb{Z} & \text { for } \quad 0 \leq k \leq n \quad \text { and } i \geq 1, \\
d_{n}^{i} \sum_{\ell=0}^{m-k} \frac{(-1)^{i}}{\left(\ell+\frac{1}{2}\right)^{i}} \in \mathbb{Z} & \text { for } 0 \leq k \leq m \text { and } i \geq 1, \\
d_{n-1}^{i} \sum_{\ell=1}^{k-m-1} \frac{1}{\left(\ell-\frac{1}{2}\right)^{i}} \in \mathbb{Z} & \text { for } m+1 \leq k \leq n \text { and } i \geq 1 .
\end{array}
$$




\section{Asymptotic behaviour}

In this section we make frequent use of Stirling's asymptotic formula

$$
n ! \sim \sqrt{2 \pi n}\left(\frac{n}{e}\right)^{n} \quad \text { as } n \rightarrow \infty,
$$

and its corollary

$$
\left(\begin{array}{c}
2 n \\
n
\end{array}\right) \sim \frac{2^{2 n}}{\sqrt{\pi n}} \quad \text { as } n \rightarrow \infty
$$

for the central binomial coefficients. (One may also use somewhat weaker but 'more elementary' lower and upper bounds

$$
\int_{1}^{n} \log x \mathrm{~d} x \leq \log (n !) \leq \int_{2}^{n+1} \log x \mathrm{~d} x
$$

for the factorial coming out from estimating integral sums of the logarithm function, with a nemesis of running into more sophisticated versions for the asymptotics and inequalities below.)

Because the rational function $R_{n}(t)$ in $(2)$ vanishes at $1,2, \ldots, n$ and at $\frac{1}{2}, \frac{3}{2}, \ldots, n-\frac{1}{2}$, the hypergeometric series (1) can be alternatively written as

$$
r_{n}=\sum_{\nu=n+1}^{\infty} R_{n}(\nu)=\sum_{k=0}^{\infty} c_{k} \quad \text { and } \quad \hat{r}_{n}=\sum_{\nu=n+1}^{\infty} R_{n}\left(\nu-\frac{1}{2}\right)=\sum_{k=0}^{\infty} \hat{c}_{k}
$$

with the involved summands

$$
c_{k}=R_{n}(n+1+k)=\frac{2^{6 n} n !^{s^{-5}} \prod_{j=0}^{6 n}\left(k+1+\frac{1}{2} j\right)}{\prod_{j=0}^{n}(n+k+1+j)^{s+1}}=\frac{n !^{s-5}(6 n+2 k+2) !(n+k) !^{s+1}}{2(2 k+1) !(2 n+k+1) ! !^{s+1}}
$$

and

$$
\hat{c}_{k}=R_{n}\left(n+\frac{1}{2}+k\right)=\frac{2^{6 n} n !^{s-5} \prod_{j=0}^{6 n}\left(k+\frac{1}{2}+\frac{1}{2} j\right)}{\prod_{j=0}^{n}\left(n+k+\frac{1}{2}+j\right)^{s+1}}
$$

strictly positive. Observe that

$$
\begin{aligned}
\frac{c_{k}}{\hat{c}_{k}} & =\frac{\prod_{j=0}^{6 n}(2 k+2+j)}{\prod_{j=0}^{6 n}(2 k+1+j)} \cdot\left(\prod_{j=0}^{n} \frac{n+k+\frac{1}{2}+j}{n+k+1+j}\right)^{s+1} \\
& =\frac{6 n+2 k+2}{2 k+1} \cdot\left(2^{-2(n+1)} \frac{\left(\begin{array}{c}
4 n+2 k+2 \\
2 n+k+1
\end{array}\right)}{\left(\begin{array}{c}
2 n+2 k \\
n+k
\end{array}\right)}\right)^{s+1} \\
& \sim \frac{6 n+2 k+2}{2 k+1}\left(\frac{n+k}{2 n+k+1}\right)^{(s+1) / 2} \text { as } n+k \rightarrow \infty .
\end{aligned}
$$

Lemma 4. For $s \geq 7$ odd,

$$
\lim _{n \rightarrow \infty} r_{n}^{1 / n}=\lim _{n \rightarrow \infty} \hat{r}_{n}^{1 / n}=g\left(x_{0}\right) \quad \text { and } \quad \lim _{n \rightarrow \infty} \frac{r_{n}}{\hat{r}_{n}}=1
$$

where

$$
g(x)=\frac{2^{6}(x+3)^{6}(x+1)^{s+1}}{(x+2)^{2(s+1)}}
$$

and $x_{0}$ is the unique positive zero of the polynomial

$$
x(x+2)^{(s+1) / 2}-(x+3)(x+1)^{(s+1) / 2} .
$$


Proof. We have

$$
\frac{c_{k+1}}{c_{k}}=\frac{\left(k+3 n+\frac{3}{2}\right)(k+3 n+2)}{(k+1)\left(k+\frac{3}{2}\right)}\left(\frac{k+n+1}{k+2 n+2}\right)^{s+1} \sim f\left(\frac{k}{n}\right)^{2} \quad \text { as } \quad n+k \rightarrow \infty,
$$

where

$$
f(x)=\frac{x+3}{x}\left(\frac{x+1}{x+2}\right)^{(s+1) / 2} .
$$

For an ease of notation write $q=(s+1) / 2 \geq 4$. Since

$$
\frac{f^{\prime}(x)}{f(x)}=\frac{1}{x+3}-\frac{1}{x}+q\left(\frac{1}{x+1}-\frac{1}{x+2}\right)=\frac{(q-3) x^{2}+3(q-3) x-6}{x(x+1)(x+2)(x+3)}
$$

and the quadratic polynomial in the latter numerator has a unique positive zero $x_{1}$, the function $f(x)$ monotone decreases from $+\infty$ to $f\left(x_{1}\right)$ when $x$ ranges from 0 to $x_{1}$ and then monotone increases from $f\left(x_{1}\right)$ to $f(+\infty)=1$ (not attaining the value!) when $x$ ranges from $x_{1}$ to $+\infty$. In particular, there is exactly one positive solution $x_{0}$ to $f(x)=1$. Notice that $0<x_{0}<1$, because $f(1)=4 \cdot(2 / 3)^{q}<1$.

The information gained and asymptotics in (11) imply that $c_{k+1} / c_{k}>1$ for the indices $k<x_{0} n-\gamma \sqrt{n}$ and $c_{k+1} / c_{k}<1$ for $k>x_{0} n+\gamma \sqrt{n}$ for an appropriate choice of $\gamma>0$ dictated by application of Stirling's formula to the factorials defining $c_{k}$ in (9) (see [2, Section 3.4] as well as the second proof of Lemma 3 in [1]). This means that the asymptotic behaviour of the sum $r_{n}=\sum_{k=0}^{\infty} c_{k}$ is determined by the asymptotics of $c_{k_{0}}$ and its neighbours $c_{k}$, where $k_{0}=k_{0}(n) \sim x_{0} n$ and $\left|k-k_{0}\right| \leq \gamma \sqrt{n}$, so that

$$
\begin{aligned}
& \lim _{n \rightarrow \infty} r_{n}^{1 / n}=\lim _{n \rightarrow \infty} c_{k_{0}(n)}^{1 / n} \\
& =\lim _{n \rightarrow \infty}\left(\left(\frac{n}{e}\right)^{(s-5) n}\left(\frac{6 n+2 k_{0}+2}{e}\right)^{6 n+2 k_{0}+2}\left(\frac{e}{2 k_{0}+1}\right)^{2 k_{0}+1}\right. \\
& \left.\times\left(\frac{n+k_{0}}{e}\right)^{(s+1)\left(n+k_{0}\right)}\left(\frac{e}{2 n+k_{0}+1}\right)^{(s+1)\left(2 n+k_{0}+1\right)}\right)^{1 / n} \\
& =\frac{\left(2 x_{0}+6\right)^{2 x_{0}+6}\left(x_{0}+1\right)^{(s+1)\left(x_{0}+1\right)}}{\left(2 x_{0}\right)^{2 x_{0}}\left(x_{0}+2\right)^{(s+1)\left(x_{0}+2\right)}} \\
& =\frac{2^{6}\left(x_{0}+3\right)^{6}\left(x_{0}+1\right)^{s+1}}{\left(x_{0}+2\right)^{2(s+1)}} \cdot f\left(x_{0}\right)^{2 x_{0}}=g\left(x_{0}\right) \text {. }
\end{aligned}
$$

It now follows from (10) that

$$
\frac{\hat{c}_{k+1}}{\hat{c}_{k}} \sim \frac{c_{k+1}}{c_{k}} \quad \text { as } \quad n+k \rightarrow \infty,
$$

so that the above analysis applies to the sum $\hat{r}_{n}=\sum_{k=0}^{\infty} \hat{c}_{k}$ as well, and its asymptotic behaviour is determined by the asymptotics of $\hat{c}_{k_{0}}$ and its neighbours $\hat{c}_{k}$, where $k_{0}=k_{0}(n) \sim x_{0} n$ and $\left|k-k_{0}\right| \leq \hat{\gamma} \sqrt{n}$. From (12) we deduce that the limits of $\hat{c}_{k_{0}(n)}^{1 / n}$ and $c_{k_{0}(n)}^{1 / n}$ as $n \rightarrow \infty$ coincide, hence $\hat{r}_{n}^{1 / n} \rightarrow g\left(x_{0}\right)$ as $n \rightarrow \infty$. In addition to this, we also get

$$
\lim _{n \rightarrow \infty} \frac{r_{n}}{\hat{r}_{n}}=\lim _{n \rightarrow \infty} \frac{c_{k_{0}(n)}}{\hat{c}_{k_{0}(n)}}=\lim _{n \rightarrow \infty} \frac{6 n+2 k_{0}+2}{2 k_{0}+1}\left(\frac{n+k_{0}}{2 n+k_{0}+1}\right)^{(s+1) / 2}=f\left(x_{0}\right),
$$

which leads to the remaining limiting relation. 


\section{Conclusion}

We choose $s=25$ and apply Lemma 4 to find out that $7 r_{n}-\hat{r}_{n}>0$ for $n$ sufficiently large, and

$$
\lim _{n \rightarrow \infty}\left(7 r_{n}-\hat{r}_{n}\right)^{1 / n}=g\left(x_{0}\right)=\exp (-25.292363 \ldots),
$$

where $x_{0}=0.00036713 \ldots$ Assuming that the odd zeta values from $\zeta(5)$ to $\zeta(25)$ are all rational and denoting by $a$ their common denominator, we use Lemma 3 and the asymptotics (3) to conclude that the sequence of positive integers

$$
a d_{n}^{25}\left(7 r_{n}-\hat{r}_{n}\right)
$$

tends to 0 as $n \rightarrow \infty$; contradiction. Thus, at least one of the numbers $\zeta(5), \zeta(7), \ldots, \zeta(25)$ is irrational.

Those who count the prime number theorem as insufficiently elementary may use weaker versions of (3), for example, $d_{n}<3^{n}$ from [5] and the choice $s=33$ instead, to arrive at the same conclusion (for the larger value of $s$, of course).

Finally, we remark that the novelty of eliminating an 'unwanted' term of $\zeta(3)$ in linear forms in odd zeta values can be further used with the arithmetic method in [10] to significantly reduce the size of $s$. Since this does not let $s$ be down to $s=9$, hence leaving the achievement 'at least one of the four numbers $\zeta(5), \zeta(7), \zeta(9), \zeta(11)$ is irrational' unchanged, we do not discuss this generalisation in greater details. We point out, however, that there are other applications of the hypergeometric 'twist-by-half' idea, some discussed in the joint papers $[6,8]$, and that a far-going extension to general 'twists' introduced by J. Sprang in [9] leads to an elementary proof of a version of the Ball-Rivoal theorem from [1] as well as to a significant improvement of the latter — see [4] for details.

\section{Acknowledgements}

I thank Stéphane Fischler, Tanguy Rivoal, Johannes Sprang and the anonymous referees for their feedback on the manuscript.

\section{References}

[1] Ball K., Rivoal T., Irrationalité d'une infinité de valeurs de la fonction zêta aux entiers impairs, Invent. Math. 146 (2001), 193-207.

[2] de Bruijn N.G., Asymptotic methods in analysis, Bibliotheca Mathematica, Vol. 4, North-Holland Publishing Co., Amsterdam, P. Noordhoff Ltd., Groningen, Interscience Publishers Inc., New York, 1958.

[3] Fischler S., Irrationalité de valeurs de zêta (d'après Apéry, Rivoal, ... ), Astérisque (2004), no. 294, 27-62, math.NT/0303066.

[4] Fischler S., Sprang J., Zudilin W., Many odd zeta values are irrational, arXiv:1803.08905.

[5] Hanson D., On the product of the primes, Canad. Math. Bull. 15 (1972), 33-37.

[6] Krattenthaler C., Zudilin W., Hypergeometry inspired by irrationality questions, arXiv:1802.08856.

[7] Rivoal T., Irrationalité d'au moins un des neuf nombres $\zeta(5), \zeta(7), \ldots, \zeta(21)$, Acta Arith. 103 (2002), 157167, math.NT/0104221.

[8] Rivoal T., Zudilin W., A note on odd zeta values, arXiv:1803.03160.

[9] Sprang J., Infinitely many odd zeta values are irrational. By elementary means, arXiv:1802.09410.

[10] Zudilin W., Arithmetic of linear forms involving odd zeta values, J. Théor. Nombres Bordeaux 16 (2004), 251-291, math.NT/0206176. 